\title{
ACOUSTIC TRANSMISSION AND REFLECTION BY A SHEAR DISCONTINUITY SEPARATING HOT AND COLD REGIONS
}

\author{
S. M. CANDEL \\ Daniel and Florence Guggenheim Jet Propulsion Center, \\ California Institute of Technology, \\ Pasadena, California 91109, U.S.A.
}

(Received 31 May 1972)

Acoustic transmission and reflection is analyzed for plane waves propagating from a hot moving medium, impinging on a plane shear discontinuity into a cold stationary region. It is shown that incident waves originating in the hot region and propagating in the flow direction are transmitted into the cold region at almost right angles to the interface. The result is employed to examine the strong side radiation of internal noise transmitted through the exhaust duct of a turbojet engine.

\section{INTRODUCTION}

The reflection of sound by an interface separating two regions in relative motion, originally examined briefly by Rayleigh [1] was subsequently treated by Rudnick [2], Keller [3], and Franken and Ingard [4]. Miles [5], in an extensive examination of the problem, corrected an erroneous interfacial kinematic condition used in the earlier work. Utilizing the properly formulated problem, Miles [5] and Ribner [6] discussed the effects of the velocity discontinuity on the transmission, reflection, and the possibility of resonance, amplification and instability of the interface.

The effect of a simultaneous temperature discontinuity was not studied in detail, however, and it is our purpose to show that a temperature discontinuity has a major effect on the direction of propagation on the transmitted waves.

A large temperature difference exists between the warm primary exhaust gases of a turbojet and the surrounding atmosphere. The results permit simple interpretation of certain observations of the internal noise radiation.

\section{ANALYSIS OF THE PROBLEM}

In conformity with references [5] and [6], consider a plane interface at $y=0$, separating two regions with distinct sound speeds and flow velocities. In region $1, y>0$, the sound speed is $c_{1}$ and the flow Mach number is $M$. Assume for simplicity that region $2(y<0)$ is stationary and has a sound speed $c_{2}<c_{1}$. The pressure fluctuations are $p_{\mathbf{i}} \exp (-i \omega t), R p_{\mathbf{r}} \exp (-i \omega t)$, $T p_{t} \exp (-i \omega t)$ for the incident, reflected and transmitted waves, respectively, and $v(x) \exp (-i \omega t)$ is the $y$-displacement of the vortex sheet. The incident wave has a normal direction $\Theta_{1}$; the reflected wave propagates in the direction $-\Theta_{1}$, and the transmitted wave in the direction $\Theta_{2}$ (see Figure 1):

$$
\begin{aligned}
& p_{\mathrm{i}}=\exp \left(-i \frac{k_{1} x \cos \Theta_{1}}{1-M \cos \Theta_{1}}-i \frac{k_{1} y \sin \Theta_{1}}{1-M \cos \Theta_{1}}\right), \\
& p_{\mathrm{r}}=\exp \left(-i \frac{k_{1} x \cos \Theta_{1}}{1-M \cos \Theta_{1}}+i \frac{k_{1} y \sin \Theta_{1}}{1-M \cos \Theta_{1}}\right),
\end{aligned}
$$




$$
p_{\mathrm{t}}=\exp \left(-i k_{2} x \cos \Theta_{2}-i k_{2} y \sin \Theta_{2}\right) \text {, }
$$

with

$$
\begin{gathered}
k_{1}=\omega / c_{1}, \\
k_{2}=\omega / c_{2}, \\
0<\Theta_{1}<\pi .
\end{gathered}
$$

All the waves have the same wavenumber in the $x$-direction; thus,

$$
\cos \Theta_{2}=\frac{\left(c_{2} / c_{1}\right)}{1-M} \frac{\cos \Theta_{1}}{\cos \Theta_{1}}
$$

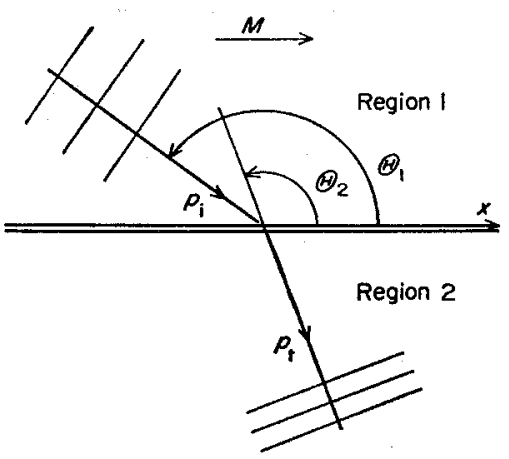

Figure 1. Geometry of the problem.

To obtain the transmission coefficient $T$ and reflection coefficient $R$, the conditions of continuity of pressure and displacement at the interface must be applied:

$$
\begin{gathered}
\left(p_{\mathrm{i}}+R p_{\mathrm{r}}\right)_{y=0_{+}}=\left(T p_{\mathrm{t}}\right)_{y=0_{-}}, \\
\left(-i \omega+U \frac{\partial}{\partial x}\right)^{2} \nu(x)=-\frac{1}{\rho_{1}} \frac{\partial}{\partial y}\left(p_{\mathrm{i}}+R p_{\mathrm{r}}\right)_{y=0_{+}}, \\
-(-i \omega)^{2} \nu(x)=-\frac{1}{\rho_{2}} \frac{\partial}{\partial y}\left(T p_{\mathrm{t}}\right)_{y=0_{-}}
\end{gathered}
$$

Equations (7), (8) and (9) lead to

$$
\begin{gathered}
1+R=T, \\
1-R=\left(\rho_{1} c_{1}^{2} / \rho_{2} c_{2}^{2}\right)\left(\sin 2 \Theta_{2} / \sin 2 \Theta_{1}\right) T,
\end{gathered}
$$

and yield the transmission coefficient:

$$
T=2 /\left(1+\rho_{1} c_{1}^{2} \sin 2 \Theta_{2} / \rho_{2} c_{2}^{2} \sin 2 \Theta_{1}\right) .
$$

The static pressures on both sides of the interface are equal and consequently the ratio $\rho_{1} c_{1}^{2} / \rho_{2} c_{2}^{2}$ reduces, for perfect gases, to $\gamma_{1} / \gamma_{2}$. It is assumed, for simplicity, that $\gamma_{1}=\gamma_{2}$.

\section{RESULTS}

When the temperature is uniform, $c_{2} / c_{1}=1$, and the transmission angle $\Theta_{2}$ is as shown on Figure 2. For an incidence $\Theta_{1}$ below the direction of total reflection, $\Theta_{1 R}=\arccos [1 /(1+M)]$, the transmission angle is complex, the transmitted waves are attenuated exponentially, and 


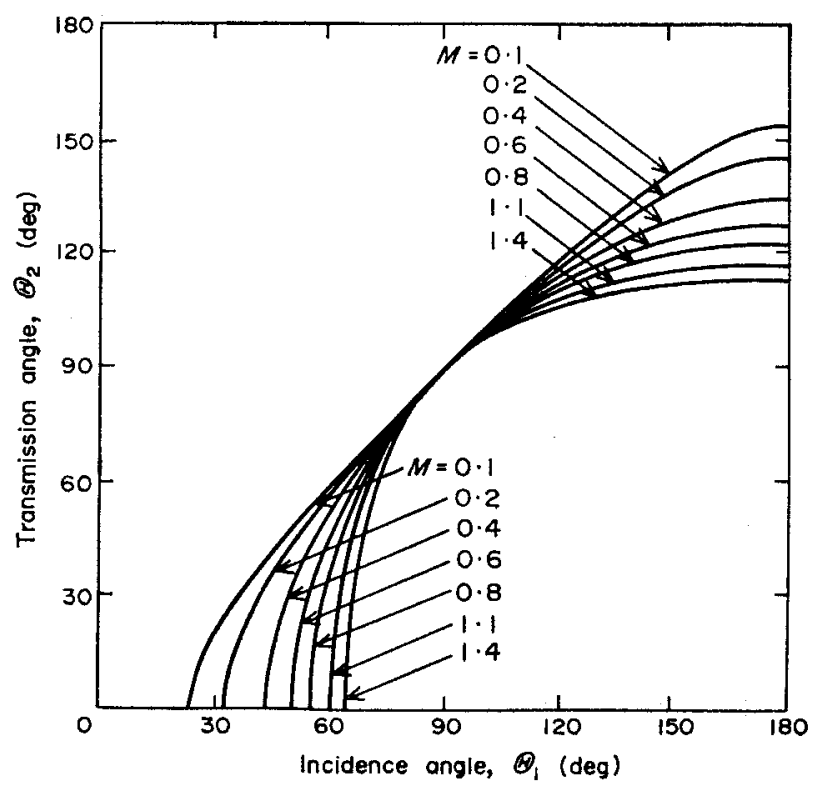

Figure 2. Transmission angle $\Theta_{2}$ for a uniform temperature across the interface. Sound speed ratio $c_{2} / c_{1}=1$.

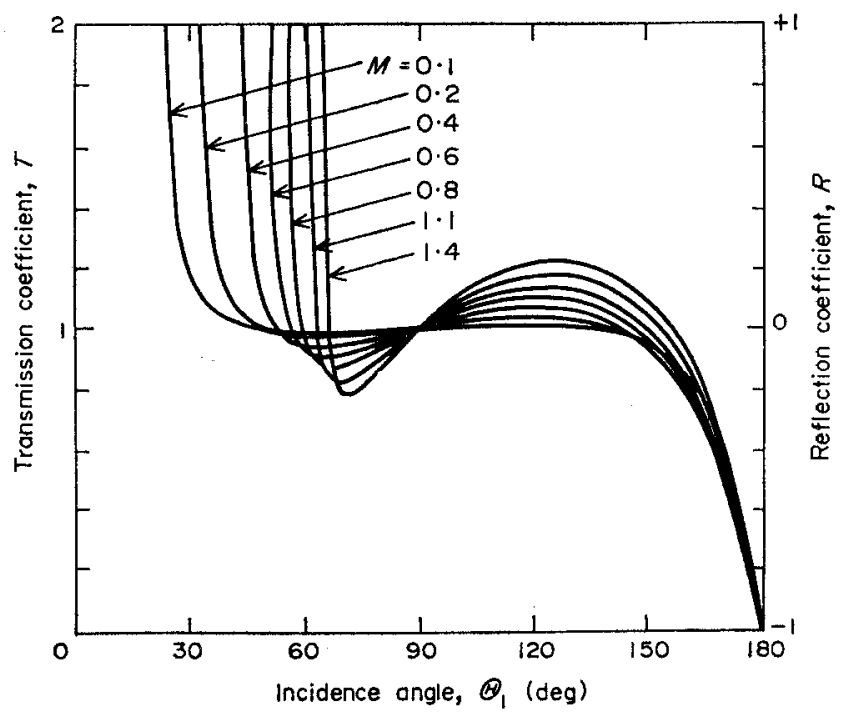

Figure 3. Transmission and reflection coefficients for a uniform temperature across the interface. Sound speed ratio $c_{2} / c_{1}=1$.

$|R|=1$. Figure 3 shows the reflection and transmission coefficients, $R$ and $T$. For normal incidence,

$$
T\left(\Theta_{1}=90^{\circ}\right)=1,
$$

and $T$ remains around this value for a large range of incidence angles.

Next consider a strong temperature difference to exist between the streams, $c_{2} / c_{1}=0 \cdot 3$. Figure 4 shows that in this case the transmission angles, $\Theta_{2}$, are strikingly different from those shown in Figure 2 for uniform temperature. When the value of $\Theta_{1}$ is between $90^{\circ}$ and $180^{\circ}$ 


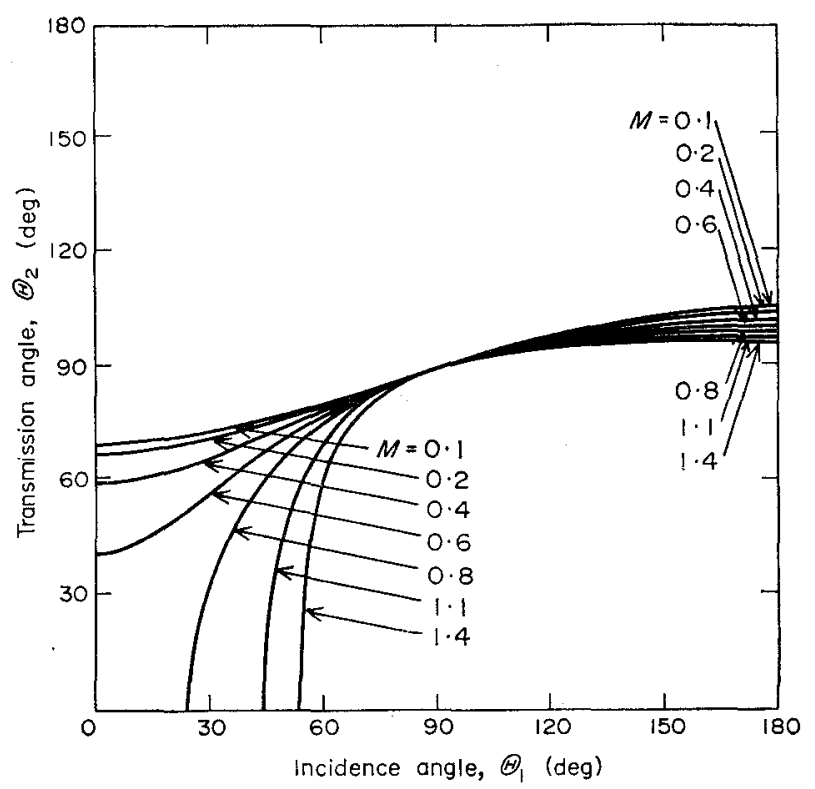

Figure 4. Transmission angle $\Theta_{2}$ for a temperature discontinuity across the interface. Sound speed ratio $c_{2} / c_{1}=\mathbf{0} \cdot 3$.

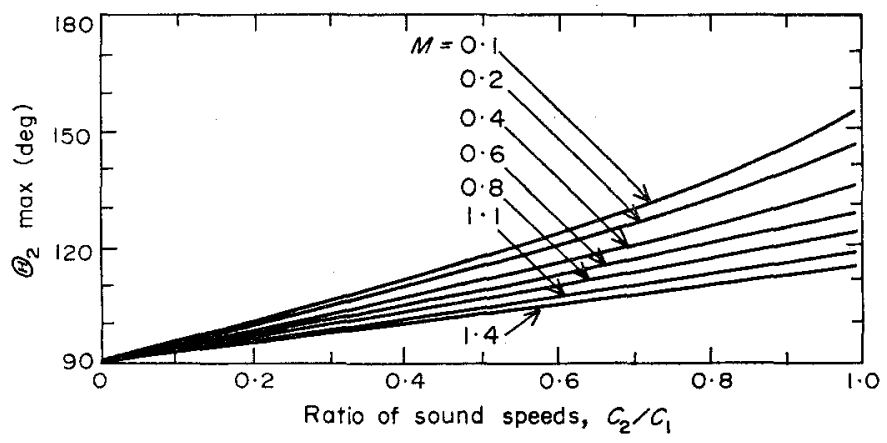

Figure 5. Maximum transmission angle $\Theta_{2 \text { max }}$. (The corresponding incidence angle is $\Theta_{1}=180^{\circ}$.)

the transmission angles crowd in a small interval near $\Theta_{2}=90^{\circ}$. For instance, when $M=0.8$, the transmission angle remains between $90^{\circ}$ and $99^{\circ}$. The transmission angle is maximum when $\Theta_{1}=180^{\circ}$; then

$$
\Theta_{2 \max }=\arccos \left(\frac{c_{2} / c_{1}}{1+M}\right)
$$

This upper bound for $\Theta_{2}$ has been represented on Figure 5 and remains below $110^{\circ}$ for $c_{2} / c_{1} \leqslant 0.6$ and $M \geqslant 0.8$. Figure 6 shows the transmission and reflection coefficients corresponding to $c_{2} / c_{1}=0 \cdot 3$. For normal incidence, the transmission coefficient is now

$$
T\left(\Theta_{1}=90^{\circ}\right)=2 /\left(1+c_{2} / c_{1}\right)
$$

and $T$ remains close to this value for a large range of angles, between $70^{\circ}$ and $150^{\circ}$.

The above results were derived for a plane configuration and an infinitely thin layer of discontinuity. However, even if the transition between regions 1 and 2 occurs continuously over a finite region, relation (6) between incidence and transmission angles remains valid. 


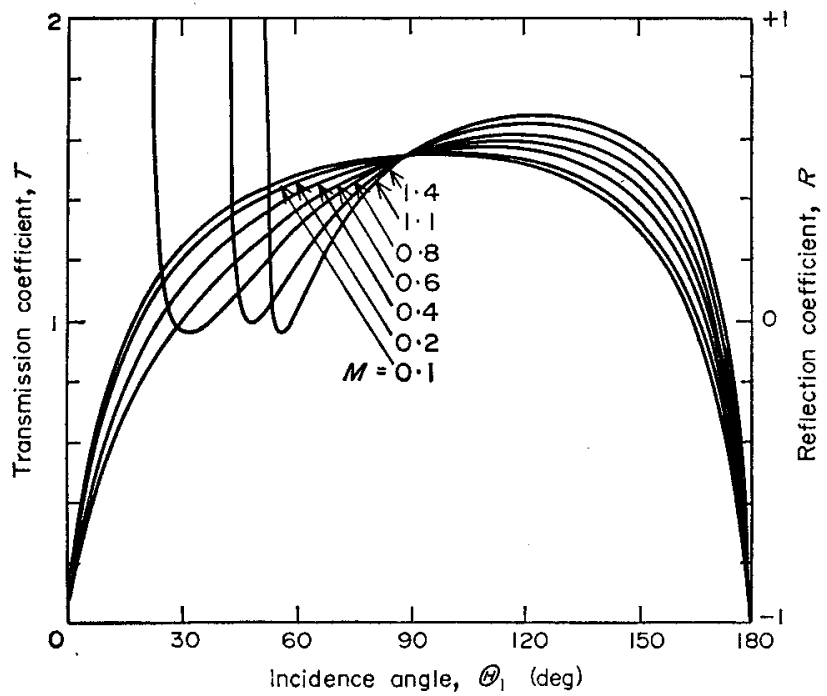

Figure 6. Transmission and reflection coefficients for a temperature discontinuity across the interface. Sound speed ratio $c_{2} / c_{1}=0 \cdot 3$.

The consequences for a turbojet engine may be inferred from the above ideal problem. The internal noise transmitted through the primary exhaust duct of a turbojet will be radiated in directions nearly at right angles to the jet axis. Such strong side radiation of internal noise has been observed by Grande [7] in a study of the JT8D engine and by Hawkins and Hoch [8] for the Olympus 593. The Olympus 593 has a particularly warm core when the afterburner is operating and also a slightly supersonic jet; the side radiation of internal noise is thus more pronounced.

\section{ACKNOWLEDGMENT}

The author wishes to express his thanks to Professor Frank E. Marble for very helpful discussion of this work.

\section{REFERENCES}

1. LoRd RAYLEIGH 1877 Theory of Sound. New York: Dover Publications. Second edition, 1945 re-issue. See volume 2, p. 133.

2. I. RUDNICK 1946 Journal of the Acoustical Society of America 17, 245-253. Acoustic transmission through a flow lamina.

3. J. B. KeLLER 1955 Journal of the Acoustical Society of America 27, 1044-1047. Reflection and transmission of sound by a moving medium.

4. P. FRANKen and U. INGARD 1956 Journal of the Acoustical Society of America 28, 126-127. Sound propagation into a moving medium.

5. J. W. Miles 1957 Journal of the Acoustical Society of America 29, 226-228. On the reflection of sound at an interface of relative motion.

6. H. S. RIBNER 1957 Journal of the Acoustical Society of America 29, 435-441. Reflection, transmission, and amplification of sound by a moving medium.

7. E. Grande 1972 Journal of the Acoustical Society of America 51, 94(A). Exhaust noise field generated in the JT3D core engine-noise floor presented by internal sources.

8. R. HAwKINs and R. HocH 1971 Communication at the 10 th International Congress of Aeronautics, Paris. Récherches sur l'émission et la reduction du bruit des propulseurs de Concorde. 